\title{
Dispersion Dynamical Magnetic Radius in Intrinsic Spin Equals the Compton Wavelength
}

\author{
Antony J. Bourdillon \\ UHRL, San Jose, CA, USA \\ Email: bourdillona@sbcglobal.net
}

How to cite this paper: Bourdillon, A.J. (2018) Dispersion Dynamical Magnetic Radius in Intrinsic Spin Equals the Compton Wavelength. Journal of Modern Physics, 9, 2295-2307.

https://doi.org/10.4236/jmp.2018.913145

Received: September 26, 2018

Accepted: November 2, 2018

Published: November 5, 2018

Copyright $\odot 2018$ by author and Scientific Research Publishing Inc. This work is licensed under the Creative Commons Attribution International License (CC BY 4.0).

http://creativecommons.org/licenses/by/4.0/

(c) (i) Open Access

\begin{abstract}
Because magnetic moment is spatial in classical magnetostatics, we progress beyond the axiomatic concept of the point particle electron in physics. Orbital magnetic moment is well grounded in spherical harmonics in a central field. There, quantum numbers are integral. The half-integral spinor moment appears to be due to cylindrical motion in an external applied magnetic field; when this is zero $\left(B_{z}^{\text {ext }}=0\right)$, the spin states are degenerate. Consider lifting the degeneracy by diamagnetism in the cylindrical magnetic field: a uniquely derived electronic magnetic radius shares the identical value to the Compton wavelength.
\end{abstract}

\section{Keywords}

Magnetic Radius, Intrinsic Spin, Compton Wavelength, Dispersion Dynamics, Stable Wave Packet, Special Relativity, Propagation, Transverse Plane, Functions of Relativistic Free Particles, Quantum Physics, Quantum Mechanics

\section{Introduction}

There are many types of science. In mathematical quantum theory, the electron is a point particle with intrinsic spin given axiomatically. This representation is unphysical because magnetostatics provides for magnetic moment $\boldsymbol{m}$ described spatially via Ampere's law so that $\boldsymbol{m}=I \boldsymbol{a}_{\boldsymbol{s}}$, where $I$ is the current flowing around a loop with surface area $\boldsymbol{a}_{\boldsymbol{s}}$. Though mathematical conclusions are occasionally so unexpected that physical hypotheses are not credible without them; in the logic of physics, a hypothesis is meaningless if it is not falsifiable [1] [2]. This restriction includes axioms $s^{1}$ since they are always "logically true" in mathematics. Here

${ }^{1}$ The hypothesis and axiom are both ideal, but the former is tested physically, while the latter is a (sometimes enabling; sometimes misleading) mathematical short-cut or deliberate abstraction. The experiment is real. 
we develop a spatial representation for intrinsic spin. It will be described on the basis of dispersion dynamics [3], summarized below. The spin was first observed in the Stern-Gerlach experiment as a quantized phenomenon. A single beam of $A g$ atoms (each having its outer electron shell with azimuthal quantum number $I$ $=0$; and therefore orbital magnetic quantum number $m_{l}=0$ ) divided into two beams under a magnetic field gradient. The result demonstrated, not a continuous distribution of magnetic moment, but quantized intrinsic spin with $m_{s}=$ $\pm 1 / 2$.

The orbital spin that is described in spherical wave mechanics by means of the magnetic quantum number $m_{b}$ is relatively unproblematic. It is described in the harmonic bases used with the Schrödinger equation. The bases describe atomic currents and associated magnetic moments that couple to magnetic intensity inside ferromagnetic and paramagnetic materials. Orbital moment is therefore a foil for exploring the less obvious intrinsic moment. Indeed, the two moments couple intimately in atomic structure, and they precess together in externally applied magnetic fields [4]. Moreover, in atoms with multiple electrons in open shells, diverse coupling schemes describe the interactions of orbital moments with intrinsic spin. The schemes are accurately represented in atomic spectroscopy. They are also significant in spin resonance and in resonance imaging.

Perhaps the greatest significance for spin lies in the Pauli exclusion principle, because spin doubles the number of states allowed for indistinguishable Fermions having states of the same orbital quantum numbers. The spinor states are sometimes represented by Pauli spin matrices [5].

Dispersion dynamics [3] [6] [7] [8] [9] is based on the formula in special relativity which contains the functional relationship between energy $E$, momentum $p$ and rest mass $m_{o}$ of a free body, $f\left(E, p, m_{0}\right)=0$. In wave mechanics, this translates to $f\left(\omega, k, m_{0}, V\right)=0$, by substitution with angular frequency in Planck's law; with wave vector in the de Broglie hypothesis; and with potential $V<0$ for a bound particle. An immediate consequence is that the product of the group velocity and phase velocity in a free particle is equal to the square of the speed of light $c^{2}$. From the simple case of the free particle, second derivatives lead to a representation of Newton's $2^{\text {nd }}$ law of motion and of forces in electromagnetic fields.

Meanwhile in special relativity, priority goes to the direction of propagation $x$, and this is represented in the stable wave packet by the two-dimensional space-time variable $X(x, t)$ that will be described below. To these dimensions are added mass, and also electronic charge when electromagnetic interaction is involved. The transverse plane is minimally relativistic, in two further dimensions, $Y(y, t)$ and $Z(z, t)$. Probability functions in these coordinates are taken subject to the normal quantization constraints commonly understood in the Bohr model of the atom [3], and in the spherical harmonics of the time independent Schrödinger equation. The constraints are subject to ubiquitous motion due to uncertainty. 
When we study spin, we need to progress beyond Newton's second law of motion. One consequence of this law is that angular acceleration of a body $\mathrm{d} L / \mathrm{d} t$ in a circulating frame is proportional to the torque $\tau$ applied, which is normal to the force $F$ in the second law. Though in an ideal couple (of forces), the net force is zero; continued application of the torque causes a body to precess about an axis of circulation. This occurs in gravitational fields, as in the gyroscope, and also in electromagnetic fields, as in spin $^{2}$. From comparisons between orbital spin and intrinsic spin in the context of the stable wave packet, it will be possible to derive a physical model that is consistent in all three phenomena: orbital spin, intrinsic spin and precessing torques [10].

\section{Dispersion Dynamics in Summary}

\subsection{Wave-Particle Duality}

The most fundamental feature of modern physics is wave-particle duality ${ }^{3}$. It is best expressed by the stable wave packet: self-evidently stable as the travelling wave group for a free particle or photon:

$$
\phi=A \cdot \exp \left(\frac{X^{2}}{2 \sigma^{2}}+X\right), \quad \text { with } X=i(\bar{k} x-\bar{\omega} t)
$$

as illustrated in Figure 1. The mean wave vector $\bar{k}$ variable and mean angular frequency $\bar{\omega}$ are stable, not only because they are mean values of a symmetric wave function; but they are guaranteed stable by respective conservation of energy and momentum; and triple guaranteed by symmetry in space-time. In the direction of propagation, $X$ is an imaginary variable that causes $\phi$ to oscillate in

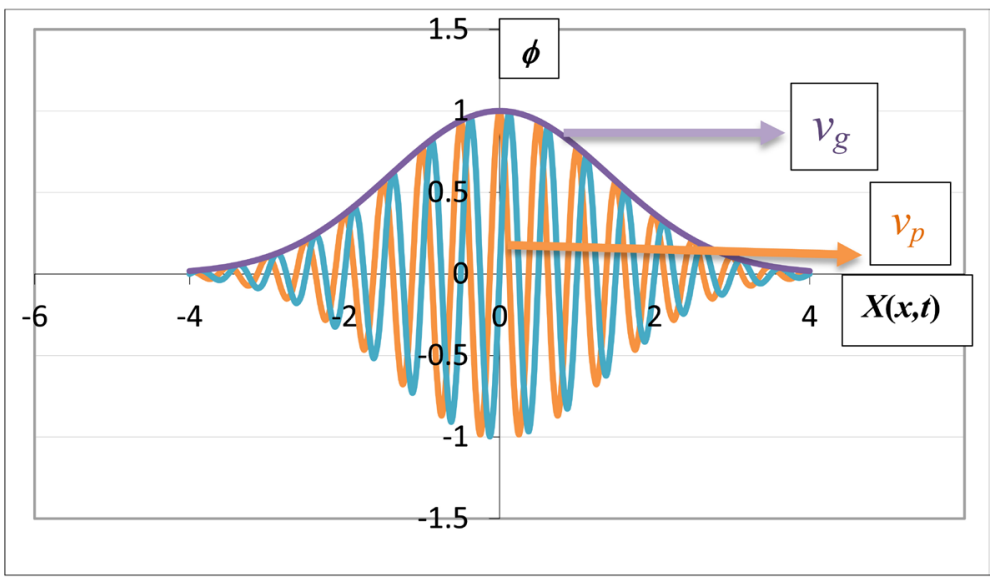

Figure 1. Stable wave packet (Equation (1)) containing envelope with group velocity $v_{g}$ (violet arrow) and real (orange) and imaginary (green) parts of the carrier wave with phase velocity $V_{p}$ (orange arrow).

${ }^{2}$ By converse, the Lorentz force is a torque on a charged particle moving across a magnetic field. The torque axis $\tau$ is proportional to the acceleration of particle angular momentum $\mathrm{d} L / \mathrm{d} t$ and is normal to both the velocity and magnetic field intensity, which in combination, are proportional causes for the torque [3].

${ }^{3}$ The information in this section has been written in greater detail elsewhere; but here the emphasis is directed, with cursory references to other specific applications of the theory. 
the complex exponential function $\exp (X)$. This describes the first part of the wave-particle duality. The other argument in the exponential function, $\exp \left(X^{2} / 2 \sigma^{2}\right)$, is real and describes the particle. Here, the denominator $\sigma$ is particular because it depends on initial conditions, but it is stable during propagation in free space as a consequence of Newton's first law of motion. The normalizing amplitude $A$ depends on the coherence $\sigma$ and, in free space, is therefore equally stable. The envelope depends on the square of $X$ which is a function of four variables. Two are already considered, so we are left with the variables $x$ and $t$ that describe the profile. Since the other variables are all stable, this profile is also stable. Following Dirac's opinion [11], it has been supposed that the wave packet is unstable; but Equation (1) enables new perspectives, including the dispersion dynamics exemplified in the following analysis.

\subsection{Solutions for the Particle Function $f\left(\omega, k, m_{0}, V\right)=0$}

From equation (1) are also derived Planck's law, $E=\hbar \omega$, the de Broglie hypothesis $\boldsymbol{p}=\hbar \boldsymbol{k}$, and several conservation rules. Solve $f\left(\omega, k, m_{0}, V\right)=0$, first for the free particle with rest mass $m_{o}$ in potential $V=0$. The relativistic Klein-Gordon equation, $\left(\square^{2}-m_{0}^{2}\right) \phi(x)=0$, operating on Equation (1) yields, for free particles, an algebraic equation in second order:

$$
\hbar^{2} \omega^{2}=\hbar^{2} k^{2} c^{2}+m_{0}^{2} c^{4}
$$

$\hbar$ being the reduced Planck constant and $c$ the speed of light. This is the same equation as is obtained from Einstein's relativistic formula, $E^{2}=p^{2} c^{2}+m_{0}^{2} c^{4}$, by substituting for energy using Planck's law and for momentum using the de Broglie hypothesis. The equation can be simplified with appropriate units $c=1$ $=\hbar$. Differentiation then gives a new result in relativity, for the product of group velocity $\mathrm{d} \omega / \mathrm{d} k[3]$, and (more obviously) phase velocity $\omega / k$ :

$$
\frac{\mathrm{d} \omega}{\mathrm{d} k} \cdot \frac{\omega}{k}=v_{g} \cdot v_{p}=1 \quad\left(=c^{2} \text { in generalized units }\right)
$$

The result is plotted in the positive quadrant of Figure 2 for the case rest mass $m_{o}=1$. The group velocity is well behaved: it tends to zero at low $k$ and to $c$ at large $k$ exactly as in the special theory of relativity ${ }^{4}$. The phase velocity is faster than the speed of light $c$ and is singular when $k \rightarrow 0$ : within this rest frame, time is Newtonian within the coherence $\sigma$. This has significance in the reduction of the wave packet during a quantum transition [3], most notably to support superconductivity in the absence of electric field. Notice that particle-like properties (including speed) are represented by the absolute value of the wave group, while wave interference and superposition are represented by the complex phase of the carrier wave.

Progressing to second derivatives, we can represent Newton's second law of motion in terms of dispersion dynamics. An important application is the Hall effect which shows negative coefficients in $\mathrm{Cu}$, n-type semiconductors, and high

${ }^{4}$ Those that calculate the speed of the electron should know which velocity they mean to calculate cf. [11]. 


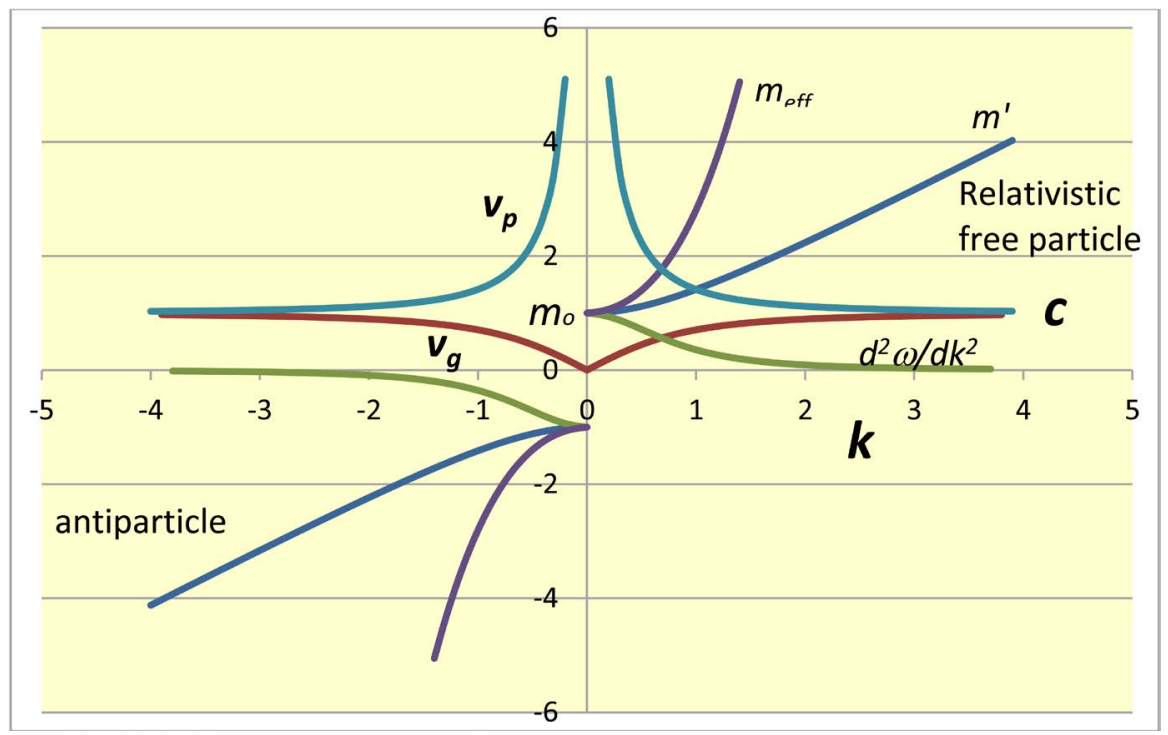

Figure 2. Functions for a free particle in Dispersion Dynamics, where $f\left(\omega, k, m_{0}\right)=0$, plotted against abscissae wave vector $k$. Ordinates are a mathematical set, being the ratios of the various physical quantities/unit values, including the energy of a relativistic free particle where $\hbar \omega=m^{\prime} c^{2}=\left(k^{2}+m_{0}^{2}\right)^{1 / 2}$ in case $m_{0}=1$ (using units $c=1=\hbar$ ); phase velocity $V_{p ;}=\omega / k$, group velocity $\mathrm{d} \omega / \mathrm{d} k$; dispersive curvature $\mathrm{d}^{2} \omega / \mathrm{d} k^{2}$; and effective mass $m_{\text {eff }}=\left(\mathrm{d} v_{g} / \mathrm{d} k\right)^{-1}$. Negative mass [12] in antiparticles is the necessary solution for the unphysical singularity when, alternately, $\hbar k=-m_{0} c$ [8]. The antiparticles are plotted with negative $k$, being the alternative representation for the Feynman-Stückelberg switching principle [13] [14], now: "an antiparticle traveling forward in time has negative momentum."

temperature superconductors; but positive coefficients in $A l$, p-type semiconductors and low temperature superconductors, all consistent with dispersion dynamics [3]. Differentiating again Equation (3):

$$
\frac{\mathrm{d}^{2} \omega}{\mathrm{d} k^{2}}=\frac{\mathrm{d} v_{g}}{\mathrm{~d} k}=\frac{\mathrm{d} v_{g}}{\mathrm{~d} p}=\left(\frac{1}{m^{\prime}}-\frac{k^{2}}{m^{\prime 3}}\right)=\frac{1}{m_{\text {eff }}}=\frac{a}{F}
$$

where $m^{\prime}$ is the relativistic mass $m_{o} /\left(1-v_{g}^{2} / c^{2}\right)^{1 / 2}$; effective mass $m_{\text {eff }}$ is as defined in the brackets; $a$ is acceleration in Newton's second law of motion corresponding to applied force $F$, such as the Lorentz force in magnetism. Notice that a negative second derivative, or curvature, causes negative effective mass and negative acceleration due to an applied Lorentz force. This is why the Hall coefficient is positive in p-type semiconductors, $A l$ metal and high temperature superconductors, where the charge carriers in these cases can only be electrons [3].

\subsection{Quantization by Harmonics}

Then Quantum physics becomes a consequence of wave motion, combined with spatio-temporal, self-interference constraints on bound states: as in spectral emissions and absorptions between quantized atomic terms. For example, without the quantization provided in the Bohr atomic model and by the Schrödinger equation, wave functions would destructively self-interfere. The energy is quan- 
tized in the harmonic basis vectors.

Two important facts are: the expected energy or relativistic mass, integrated in time over the packet in Equation (1), is equal to $\hbar \bar{\omega}$; while the expected momentum, integrated over space, is equal to $\hbar \bar{k}$. This description is a physical, non-axiomatic description of quantization. These conditions are set by initial and final states. We will see how the model applies to intrinsic spin. Notice that in the calculation of spectral lines, such as the Lyman $\alpha$ for the hydrogen atom, rest mass energy cancels between the ground and excited terms.

As another physical description, the Uncertainty Principle can be derived from Equation (1) by Fourier transforms [3]. The derivation accounts for negative "uncertainties", as occurs in Fresnel diffraction in the near field.

\subsection{Velocity}

Dirac's calculation for the speed of the electron found it equal to $c$ [11]. We understand that calculation to fail by relativity which implies infinite mass-energy at that speed, as also does his "jitter". By contrast, the phase velocity, $\omega / k$, brings clarity. It breaks the light barrier. Can it be measured? Yes, as the inverse of the group velocity, or as the ratio of energy to momentum. But is it real? No: for spatio-temporal reasons the energy in the packet is $\hbar \bar{\omega}$, but the wave function is complex so that energy is carried by the absolute group, $\int \phi^{*} \phi \mathrm{d} \tau$; not by the phase. What has to be understood is how peaks in the complex carrier wave-whether real or imaginary-appear, grow, pass through the group, and disappear, as if elastic. Their importance lies in superposition and interference.

\subsection{Highly Relativistic and Non-Relativistic Approximations}

Two extreme regimes are commonly identified: relativistic when $p \gg m_{o} c$, and non-relativistic when $p \ll m_{o} c$.

Relativistically, at high $k \gg m_{o}$, (simplified units) both the group velocity and phase velocity tend to the speed of light: $V_{g}, V_{p} \rightarrow c$, as in the massless photon travelling in free space. Then $\mathrm{d} \omega / \mathrm{d} k=\omega / k=v^{\prime} \lambda$, the product of oscillational frequency $v^{\prime}$ with wavelength. Conductance depends on the group velocity.

At low $k \ll m_{o}$, non-relativistic values approximate:

$$
E=m_{o}\left(1+p^{2} c^{2} /\left(m_{o}^{2}\right)\right)^{1 / 2} \approx m_{o}+p^{2} / 2 m_{o}
$$

In classical mechanics, the mass energy is ignored as constant in mechanical or chemical changes, as it is in Schrödinger's equation which is likewise non-relativistic. Moving on from the free particle, when the potential $V<0$ is included, the Schrödinger eigenvalue $\varepsilon$ corresponds with the result of the virial theorem, so that the expectation value for $\langle|V|\rangle \approx-2\left\langle\left|p^{2} / 2 m_{0}\right|\right\rangle$ (Figure 3) and:

$$
\varepsilon \approx\left\langle\left|\hbar^{2} k^{2} / 2 m_{o}\right|\right\rangle
$$

using the simplified units previously described. The kinetic energy for the free particle is positive; while the eigenvalue in a potential $V<0$ is similar, but negative. 


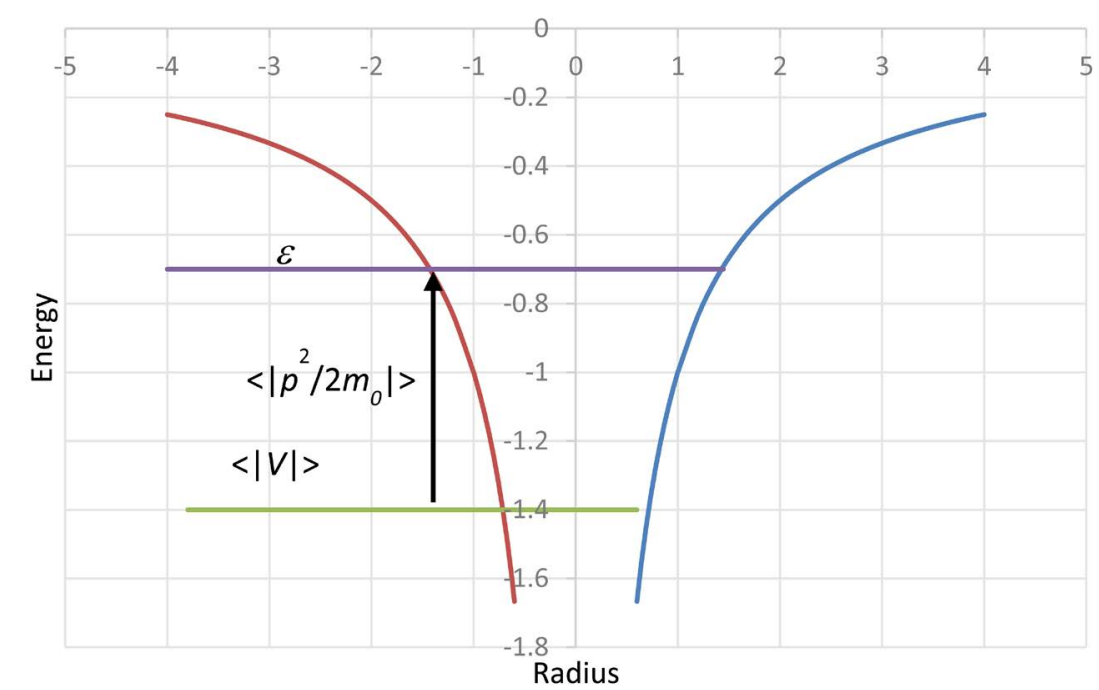

Figure 3. Following the Virial theorem, the expectation value for the potential energy is double the expectation for the negative of the kinetic energy on, for example, an electron bound by a Coulomb potential. The difference is the eigenvalue $\varepsilon$. In Dispersion Dynamics, the non-relativistic group velocity is approximately half the ratio of the energy/momentum, $v_{g} \rightarrow \varepsilon / 2 p$, as in classical mechanics. However, when $k \gg m_{o}$, then $v_{g} \rightarrow$ $k / \omega \approx c$.

Whether free or bound, the group velocity is given by:

$$
v_{g}=\frac{E-m_{o}}{2 p}=\frac{\omega-m_{o}}{2 k}
$$

or $\mathcal{E} / 2 p$ in the system of Schrödinger. This is the velocity that is proportional to the Lorentz force of magnetism for a charged particle moving in a magnetic field, as in Hall effect measurements mentioned earlier.

\section{Intrinsic Spin}

\section{Dimensions}

Intrinsic spin is a physical quantity. Our new starting point is the stable wave packet of Equation (1) which is 2-dimensional in time and space. To these are added relativistic mass and electromagnetic charge. It is well known that the transverse plane is weakly relativistic, for example after application of a transverse force, the transverse component of the energy:

$$
E_{y}=\frac{p_{y}^{2}}{2 p_{x}}
$$

where momentum in the propagation direction replaces rest mass in the classical expression for kinetic energy. This fact shows that the transverse wave motion is dependent on the propagation. We assume therefore that the transverse components and propagation components of the 4-dimensional wave must be related in phase. The force transforms the propagation direction: 


$$
p_{x}^{\prime} \rightarrow p_{x} \hat{\boldsymbol{i}}+p_{y} \hat{\boldsymbol{j}}
$$

as indicated by the Cartesian unit vectors. Components of angular momentum are illustrated in Figure 4. Since the momentum change is normal to an angular momentum, the change is accompanied by a torque [10] operating on the transverse plane. This is due to a law of conservation in angular momentum and results in spin precession. In the absence of applied external magnetic intensity in the vertical direction $B_{z}^{e x t}=0$, presume $\omega_{z}=0$, i.e. for a plane wave in free space. The spin states are degenerate. When $B_{z}^{\text {ext }}>0$, presume that $\omega_{z}$ increases by induction, as in normal diamagnetism and in the Meissner-Ochsenfeld effect in superconductors. The degeneracy is then lifted and spin up states split from spin down. (The spin current does not decay; it is resistanceless. Under the diamagnetic hypothesis, spin in a Fermion is superconductive at room temperature, lacking a critical temperature.)

In the case of the $\mathrm{Ag}$ atoms observed in the Stern-Gerlach experiment, the origin for the magnetic quantization is due, not to the central atomic potential, but to diamagnetic Bohr orbits conceived, not at the atomic scale, but in an electron scale in the diamagnetic response to $B_{z}$. Without quantization the orbits would self-interfere and self-distruct, so the fact that $m_{s}= \pm 1 / 2$ implies that the diamagnetic Bohr orbit diameters are restricted.

In principle, a free beta ray is restricted in the same way. An experimental observation of splitting would indicate localization within the wave group because the splitting might not be uniquely quantized if it depends on the vagary in the

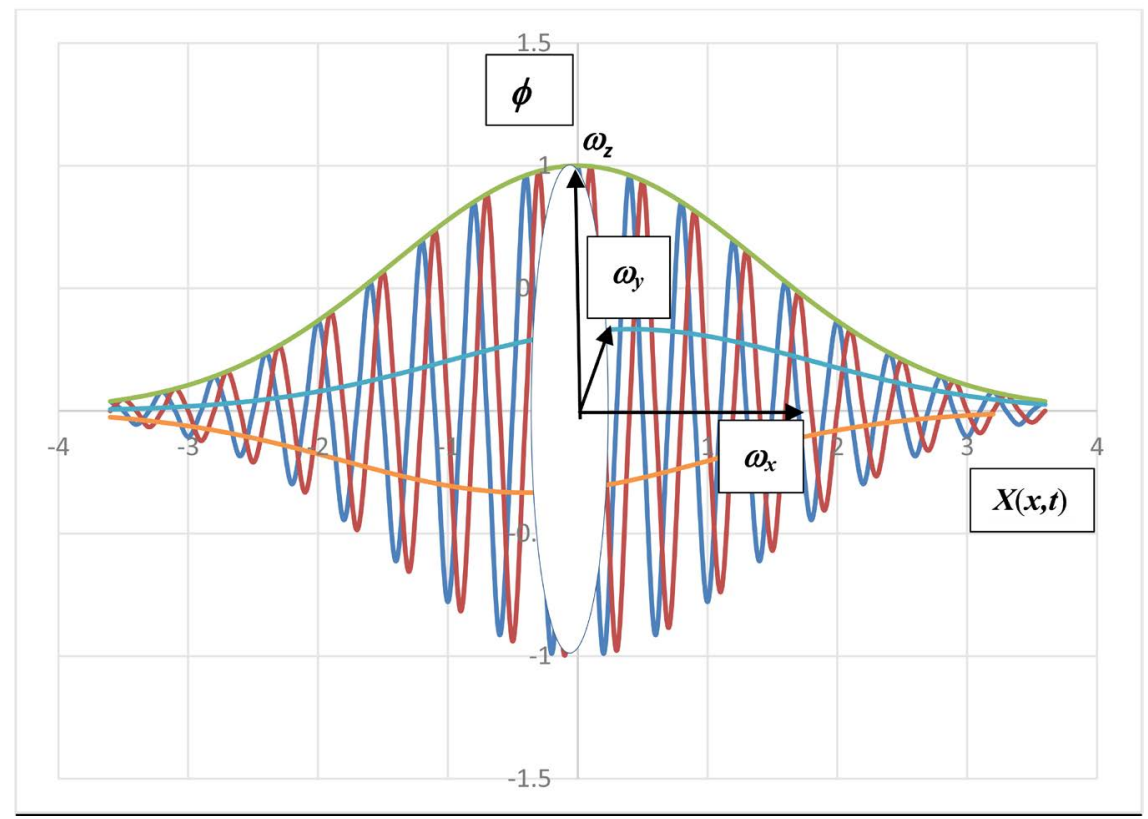

Figure 4. Wave packet showing components of angular momentum: $\omega_{x}$ in the horizontal propagation direction; and in the transverse plane $\omega_{y}$ and $\omega_{z}$. In this particular packet, the transverse plane coincides with a circle through the vertical, viewed obliquely. Absolute values of the real (dark blue) and imaginary parts (brown) are projected vertically (green line) and in the horizontal plane (light blue and orange). 
volume of the wave packet extended by its coherence $\sigma$. Notice however that, whereas a beta ray is less restricted than the atomic orbit in $\mathrm{Ag}$, Stokes' theorem applies in an evaluation of the moment in a travelling wave group, and that its electronic charge is normalized through the amplitude $A$ in Equation (1). We will return to this after finding the magnetic radius.

More generally, there are further features that delineate the nature of intrinsic spin. Firstly, the spin and is independent of wavevector $k$, but depends on mass ( $\omega$ in simplified units) when in the non-relativistic regime. Secondly, the induced currents in atomic beams imply a magnetic moment $\mu$ with electron magnetic radius $r_{m}$ :

$$
\mu=I a_{s}=I \pi r_{m}^{2}
$$

Meanwhile in the transverse plane, suppose that any angular frequencies are in phase with the frequency in the direction of propagation $x$, so that $\omega=\omega_{x}=\omega_{y}$ $=\omega_{z}$. These angular frequencies are coherent because of intersecting overlaps on the two-dimensional planes that are normal to them. (The frequencies are independent of mass components (Equation (7)) in angular momentum.)

These frequencies determine the current, so that:

$$
\mu=\frac{e \omega}{2 \pi} \pi r_{m}^{2}=\mu_{B} g_{s} m_{j}
$$

where $\mathrm{e}$ is the electron charge; $\mu_{B}=e \hbar / 2 m_{e}$ is the Bohr magneton with electron mass $m_{e} \sim m_{o}$; and $g_{s} \approx 2$ is the gyromagnetic ratio which makes the moment of the spinor approximately integral in $\mu_{B}$ when the spinor is half integral. The magnetic electron radius is defined when:

$$
\frac{\mu}{\mu_{B}}=1=\frac{e \omega}{2 \pi} \cdot \pi r_{m}^{2} \cdot \frac{2 \omega}{e c^{2}}
$$

by substituting $\hbar \omega=m_{e} c^{2}$, while $I=e \omega / 2 \pi$. Then the magnetic electron radius,

$$
r_{m}=c / \omega=3.86159323(35) \times 10^{-13}
$$

A value that is identical to the electron Compton wavelength $\lambda_{e}[15]$, and therefore $\alpha$ times the Bohr radius $a_{o}$ where $\alpha$ is the fine structure constant. The value is also $\alpha^{-1}$ times the classical electron radius $r_{e}$ that is calculated by supposing the mass of the electron is all electrostatic energy. The magnetic radius provides a limiting size for magnetic moment due to intrinsic spin that owes to a cylindrical applied field intensity.

The derivation applies to excited states of $A g$ where the angular momentum $I>0$, as it does to atoms having multiple electrons in Russell-Saunders, L-S, spin-orbit coupling; or in J-J coupling etc. Returning to Equation (12), the magnetic radius is a limiting value when the current is given by $e \omega / 2 \pi$. A smaller current that is spread over a larger radius might yield the same moment consistent with Stokes' theorem. However, such variation in radius would break the requirement for quantization in both wave phenomena and in magnetization: the wave function, where it repeats, must not self-destruct; and concomitantly, 
the magnetization is itself quantized as demonstrated by Stern and Gerlach. It therefore appears that the magnetic radius is fixed by quantization and describes the quantized size of the electron, i.e. less than two orders of magnitude shorter than the Bohr radius for the atom, $\lambda_{e}=\alpha a_{o}$. Atomic spin-orbit coupling then becomes the interaction of a localized electron with an atomic wave function. The explanation represents logically-pure, quantum physics.

An extension of the method (Equations (10)-(12)) shows, within an order of magnitude, that:

$$
\frac{\mu}{\mu_{B}} \sim \frac{v_{p} r_{m}}{v_{g} a_{0}}
$$

the numerator being due to phase harmonics in the cylindrical symmetry of $\boldsymbol{B}$ about the localized electron; the denominator to real charge current about the spherical atomic field (Equation (9)). The former is on particle scales; the latter on atomic scales. These scales physicalize the mathematical short-cut implicit in its idea of a point particle.

Notice that, in terms of wave-particle duality, $\omega$ is a wave property dependent on energy and equal to relativistic mass (using simplified units); $k$ is a particle property dependent on momentum, tending to zero in the rest frame where it is opposed by vibrational uncertainty. Quantized energy depends on $k$ in the circumference for Bohr model for the atom, or in the time independent Schrödinger equation; Equation (1) describes a probability amplitude for finding an electron with finite size and magnetic radius $c / w$.

The derivation described here for intrinsic spin takes evidence from dominant data obtained from atomic and chemical physics, but it extends to isospin in atomic nuclei and magnetic moments in elementary particles. The same transverse motion exists there with similar outcomes on different scales. Likewise, uncharged mesons and hadrons contain charged quarks and have magnetic moments [15]. Less is known about the neutrino [16]. Furthermore, any theory for intrinsic spin should provide a physical explanation for Pauli exclusion: here it is represented by indegeneracy of Fermionic states, consequential on residual ambient magnetism. If otherwise, uncertainties in angular momentum of degenerate states would cause those states having identical orbital and spin quantum numbers to interfere destructively. Bosons, by contrast, are not so constrained because their wave functions are real-notably in the photon-so that phases can lock coherently onto external influence. Interference is then constructive. Superconducting Cooper pairs, containing both chiralities in phase $\left(e^{k}+e^{-k}\right)$, do the same.

\section{Conclusion}

Intrinsic spin is often described by a supposedly physical axiom in a mathematical construct of possible worlds. Orbital magnetic moment from wave functions in a central field is comparatively unproblematic. By contrast, intrinsic spin states are degenerate in zero field, so that lifting of degeneracy by a cylindrical magnetic field implies diamagnetism with a magnetic electronic radius. We use the stable wave packet and dispersion dynamics to derive, independently, a value 
that turns out identical to the Compton wavelength.

\section{Conflicts of Interest}

The author declares no conflicts of interest regarding the publication of this paper.

\section{References}

[1] Popper, K.R. (1959) The Logic of Scientific Discovery. Hutchinson.

[2] Popper, K.R. (1982) Quantum Theory and the Schism in Physics In: Bartley III, W.W., Ed., Hutchinsonn.

[3] Bourdillon, A.J. (2017) Dispersion Dynamics in the Hall Effect and Pair Bonding in $\mathrm{HiT}_{\mathrm{c}}$. Nova science, NY.

[4] Woodgate, G.K. (1970) Elementary Atomic Spectra. McGraw-Hill, or Other Introductory Text.

[5] Ziman, J.M. (1969) Elements of Advanced Quantum Theory. Cambridge.

[6] Bourdillon, A.J. (2012) A Wave Group for Entanglement, Linking Uncertainties in Time and Space. Journal of Modern Physics, 3, 290-296.

https://doi.org/10.4236/jmp.2012.33041

http://www.scirp.org/journal/jmp

[7] Bourdillon, A.J. (2013) A Travelling Wave Group II: Antiparticles in a Force Field. Journal of Modern Physics, 4, 705-711. https://doi.org/10.4236/jmp.2013.46097 http://www.scirp.org/journal/jmp

[8] Bourdillon, A.J. (2014) A Travelling Wave Group III-Consistent with QED. Journal of Modern Physics, 5, 23-28. https://doi.org/10.4236/jmp.2014.51003 http://www.scirp.org/journal/jmp

[9] Bourdillon, A.J. (2017) Voids in the Hall Effect; Excitons in $\mathrm{HiT}_{\text {c. Journal of Mod- }}$ ern Physics, 8, 483-499. https://doi.org/10.4236/jmp.2017.84031

[10] Feynman, R.P., Leighton, R.B. and Sands, M. (1963) The Feynman Lectures on Physics. Addison-Wesley, see Vols I 20-8, II 34-4.

[11] Dirac, P.A.M. (1958) The Principles of Quantum Mechanics. 4th Edition, Oxford.

[12] Villata, M. (2011) CPT Symmetry and Antimatter Gravity in General Relativity. Europhysics Letters, 94, 20001.

[13] Feynman, R.P. (1949) The Theory of Positrons. Physical Review, 76, 749-769. https://doi.org/10.1103/PhysRev.76.749

[14] Stückelberg, E.C.G. (1941) Remarque à propos de la creation de paires de particules en théorie de relativité [A Remark on Particle Pair Creation in the Theory of Relativity]. Helvetica Physica Acta, 14, 588-594.

[15] Aguilar-Benitez, M., Barnett, R.M., Caso, C., Conforto, G., Crawford, R.L., Cutkosky, R.E., Eichler, R.A., Eidelman, S., Groom, D.E., Hagiwara, K., Hayes, K.G., Hernandez, J.J., Hikasa, K., Höhler, G., Kawabata, S., Manley, D.M., Montanet, L., Morrison, R.J., Oliver, K.A., Porter, F.C., Roos, M., Schindler, R.H., Shrock, R.E., Stone, J., Törnqvist, N.A., Trippe, T.G., Wohl, C.G., Yost, G.P., Technical Associates Armstrong, B., Gieselmann, K. and Wagman, G.S. (1992) Particle Properties Data Booklet, June 1992, American Institute of Physics, from the Review of Particle Properties. Physical Review Data, D45.

[16] Balantikin, A.B. (2006) Limits on Neutrino Magnetic Moments. Proceedings on the Origin of Matter and the Evolution of Galaxies (OMEGOS), Japan.

ArXiv:hep-ph/0601113

https://doi.org/10.1063/1.2234393 


\section{Glossary of Symbols in Order of Appearance}

\begin{tabular}{|c|c|}
\hline$B_{z}^{e x t}$ & z-component of magnetic field \\
\hline$m$ & magnetic moment \\
\hline$I$ & current flowing around a loop \\
\hline$a_{s}$ & surface area vector normal \\
\hline 1 & azimuthal quantum number \\
\hline$m_{1}$ & orbital magnetic quantum number \\
\hline$m_{s}$ & spin magnetic quantum number \\
\hline$E$ & energy \\
\hline$p$ & momentum \\
\hline$m_{o}$ & rest mass \\
\hline$\omega$ & angular frequency, $\bar{\omega}$ mean \\
\hline$k$ & wave vector, $\bar{k}$ mean \\
\hline$V$ & potential \\
\hline c & speed of light \\
\hline$X$ & space-time variable (propagation direction) \\
\hline$x$ & coordinate in direction of propagation \\
\hline$t$ & time coordinate \\
\hline$Y, y, Z, Z$ & corresponding transverse coordinates \\
\hline $\mathrm{d} L / \mathrm{d} t$ & angular acceleration \\
\hline$\tau$ & torque \\
\hline$F$ & force \\
\hline$\phi$ & wave function \\
\hline$\sigma$ & coherence \\
\hline$\hbar$ & reduced Planck constant \\
\hline$\square$ & d'Alembertian operator \\
\hline$V_{g}$ & group velocity $=\mathrm{d} \omega / \mathrm{d} k$ \\
\hline$V_{p}$ & phase velocity $=\omega / k$ \\
\hline$m_{e f f}$ & effective mass \\
\hline$a$ & acceleration \\
\hline$m^{\prime}$ & relativistic mass \\
\hline$v^{\prime}$ & oscillational frequency $=\omega / 2 \pi$ \\
\hline$\varepsilon$ & eigenvalue \\
\hline$p_{x} p_{y} p_{x}$ & components of momentum \\
\hline$\omega_{x}, \omega, \omega_{z}$ & components of angular frequency \\
\hline$r_{m}$ & electron magnetic radius \\
\hline$\mu$ & magnetic moment \\
\hline$e$ & electronic charge \\
\hline$\mu_{B}$ & Bohr magneton \\
\hline$g_{s}$ & gyromagnetic ratio \\
\hline$\lambda_{e}$ & Compton wavelength \\
\hline$a_{o}$ & Bohr radius \\
\hline & fine structure constant \\
\hline
\end{tabular}


$L \quad$ total orbital quantum number

$S$ total spin quantum number

$J \quad$ total magnetic quantum number 